\title{
Factores socioculturales y ergoambientales: su impacto en la satisfacción laboral de las instituciones de educación superior
}

\author{
María Teresa Escobedo Portillo \\ Universidad Autónoma de Ciudad Juárez \\ Luis Cuautle Gutiérrez \\ Gabriela Sánchez Bazán \\ Judith Cavazos Arroyo \\ Universidad Popular Autónoma del Estado de Puebla \\ Aurora Máynez Guaderrama \\ Universidad Autónoma de Ciudad Juárez
}

\section{Resumen}

Se propone un modelo conceptual relativo a la satisfacción laboral en las instituciones de educación superior. Para ello, se realizó una extensiva revisión de la literatura relacionada con los constructos satisfacción laboral, factores socioculturales y factores ergoambientales, y se identificaron sus variables incidentes. Se recomienda aplicar de forma empírica este modelo, utilizando para su análisis los modelos de ecuaciones estructurales.

\section{Palabras clave}

Educación superior, factores ergoambientales, factores socioculturales, modelo conceptual, satisfacción laboral. 


\title{
Sociocultural and ergo-environmental factors: their impact on job satisfaction in institutions of higher education
}

\author{
María Teresa Escobedo Portillo \\ Universidad Autónoma de Ciudad Juárez \\ Luis Cuautle Gutiérrez \\ Gabriela Sánchez Bazán \\ Judith Cavazos Arroyo \\ Universidad Popular Autónoma del Estado de Puebla \\ Aurora Máynez Guaderrama \\ Universidad Autónoma de Ciudad Juárez
}

\begin{abstract}
A conceptual model with regard to job satisfaction in institutions of higher education is proposed. An extensive review of the literature concerned to concepts about job satisfaction or sociocultural and ergo-environmental factors was conducted and their incidental variables identified. It is recommended to apply empirically this model using the structural equations models for its analysis.
\end{abstract}

\section{KEY WORDS}

Higher education, ergo-environmental factors, cultural factors, conceptual model, job satisfaction. 


\section{Introducción}

Las instituciones de educación superior (IES) en México han tratado de satisfacer sus objetivos en materia de recursos humanos, investigación científica y desarrollo social, teniendo presente las metas empresariales exigidas en organizaciones privadas (Moreno, Cepeda y Romero, 2004). Las universidades actuales se convierten cada vez más en corporaciones abiertas y multinacionales, en sitios de producción de nuevos conocimientos de acuerdo con las necesidades colectivas (Brunner, 1990). Esto ha provocado que el sistema de educación superior tenga relevancia en los nuevos procesos de productividad y de competitividad, y se ha exigido que las instituciones que conforman el mencionado sistema se dirijan a la evaluación institucional como uno de los mecanismos más aceptables para el mejoramiento de la excelencia universitaria (Rueda, Elizalde y Torquemada, 2003), estableciendo criterios para evaluar los insumos, los procesos y los productos que generan.

Por su relación directa con los egresados, el recurso humano que conforma a las IEs en México representa uno de sus principales intereses (Marginson, 2009). En consecuencia, evaluar la satisfacción laboral (sL) del personal se vuelve un desafío para las universidades (Locke, 1978), pues constituye un estado emocional positivo que refleja una respuesta afectiva hacia el trabajo (Belfield y Harris, 2002), y se enmarca dentro del estudio de las actitudes hacia el trabajo. Todas las IEs tienen la necesidad de determinar en qué medida sus empleados están alcanzando sus objetivos, analizar las condiciones en que se producen sus logros y los factores que han influido positiva o negativamente en los mismos, con el fin de establecer las acciones que deben realizarse para garantizar el cumplimiento efectivo y permanente de su misión (González, Hernández de Rincón y Hernández, 2007).

Para que las IEs alcancen sus objetivos en materia de sL de su personal se propone determinar y analizar las variables relativas a los factores socioculturales (FSC) y ergoambientales (FEA) que pueden repercutir en la sL de su personal académico. Para ello resulta indispensable revisar las investigaciones realizadas en las que se incluyan los factores mencionados, lo cual proporcionará la información requerida para proponer un modelo conceptual que permita evaluar la relación entre la sL y los factores socioculturales y ergoambientales. 


\section{Marco teórico}

\section{Componentes del comportamiento organizacional}

Para hablar de sL hay que detallar la disciplina a la que pertenece: el comportamiento organizacional (co), campo que estudia la forma en que los sujetos, los grupos y el contexto inciden en el comportamiento de las personas dentro de las organizaciones, y cuyo objetivo es lograr la eficiencia en las actividades de la empresa (Robbins, 1996). Para Forehand (1964, cit. en Robles et al., 2005), el co se forma con una serie de características propias de cada organización, las cuales la distinguen de otras firmas; son relativas al tiempo e influyen en la conducta de los trabajadores.

Existen temas fundamentales dentro del co como la motivación, la comunicación interpersonal, el comportamiento y el poder del líder, la estructura y los procesos de grupo, el aprendizaje, el desarrollo y la percepción de actitudes, los conflictos, los procesos de cambio, el diseño del trabajo y la tensión laboral (Herzberg, 1966; Miner, 2003) (cuadro 1).

\section{Disciplinas del comportamiento organizacional}

Dentro del campo del co intervienen disciplinas como la psicología, la sociología, la antropología y la psicología social (Robbins y Judge, 2009). La psicología actúa directamente sobre el individuo, y el resto de las áreas lo hace sobre los grupos y las estructuras de las organizaciones. Para algunos académicos (Bersanelli, 1979; Robbins y Judge, 2009), la psicología recoge hechos sobre la conducta y la experiencia, y los organiza sistemáticamente elaborando teorías para su comprensión, las cuales ayudan a conocer y explicar el comportamiento de los seres humanos y, en ocasiones, incluso a predecir sus acciones futuras, para poder intervenir en ellas. De acuerdo con Gilbert (1997), la sociología estudia los comportamientos y estructuras de los individuos y la relación con sus semejantes, apoyándose en técnicas de investigación interdisciplinarias dentro de la historia y la cultura en que nacen y se desarrollan, y su objetivo es encontrar tendencias en el comportamiento humano compartido. Por su parte, Burgos (2005) comenta que la antropología comparte el objeto de estudio con ciencias como la psicología, la historia, la economía, la biología y la política, 


\section{Cuadro 1. Componentes del comportamiento organizacional}

\begin{tabular}{lll}
\hline AutoRES Y AÑo & Componente & Descripción \\
Mitchell (1997) y Gor- & Motivación & Procesos que toman en cuenta la \\
don (1997) & & intensidad, dirección y esfuerzo del \\
& individuo hacia el logro de una meta. \\
& Se compone de tres elementos clave: \\
& intensidad, dirección y persistencia.
\end{tabular}

Jones (2008) Comunicación inter- Propone una comunicación directa personal entre los altos mandos y los niveles subsecuentes.

Ivancevich, Konopaske Comportamiento y Se refiere a los aspectos y actitudes y Matteson (2006) poder del líder que debe tener el director general para facilitar el equilibrio de los empleados tanto en el trabajo como en su vida privada.

Robbins y Judge Estructuras y procesos Enfatizan aspectos relativos a la con(2009) de grupo formación de un grupo o equipo de trabajo, la distribución de las obligaciones y la definición del liderazgo en la tarea.

Gordon (1997) Aprendizaje Describe cómo las experiencias pasadas, el conocimiento y la información que el individuo adquiere durante las etapas de su vida influyen en la descripción y diagnóstico de las cosas o situaciones.

Ballesteros (1994) y Desarrollo y percep- Proceso activo para percibir la realiJones (1990) ción de actitudes dad e interpretarla, para después organizarla de manera reflexiva. 


\section{Cuadro 1. Componentes del comportamiento organizacional (Continuacion)}

\begin{tabular}{|c|c|c|}
\hline AUTORES Y AÑO & COMPONENTE & DESCRIPCIÓN \\
\hline Fuentes (2001) & Conflictos & $\begin{array}{l}\text { Tipos y formas de solucionar con- } \\
\text { flictos, en eventos como la toma de } \\
\text { decisiones respecto a proyectos, con- } \\
\text { trataciones, etc. Acciones necesarias } \\
\text { para contar con la mejor solución } \\
\text { para la empresa, los trabajadores y } \\
\text { los clientes. }\end{array}$ \\
\hline Cisneros (2006) & Procesos de cambio & $\begin{array}{l}\text { Abarca todas las actividades dirigidas } \\
\text { al mejoramiento de la organización y } \\
\text { al compromiso de la administración. } \\
\text { Este proceso de cambio ocurre cuan- } \\
\text { do el personal se involucra de mane- } \\
\text { ra total. Para que el cambio se realice } \\
\text { con éxito, además del compromiso } \\
\text { de la empresa se deben aprovechar } \\
\text { todas las oportunidades. }\end{array}$ \\
\hline Wang et al. (2009) & Tensión laboral & $\begin{array}{l}\text { Forma en que se involucra tanto el } \\
\text { supervisor como el empleado para es- } \\
\text { tablecer las tareas y metas, y cumplir } \\
\text { con los objetivos planteados. Analiza } \\
\text { el grado de presión que ejerce la em- } \\
\text { presa o el superior a cada empleado y } \\
\text { cómo afecta en la salud de este. }\end{array}$ \\
\hline
\end{tabular}

Fuente: Elaboración propia.

por mencionar algunas; asimismo señala que se clasifica en varias ramas, entre las que se incluyen la antropología física, la antropología biológica, la antropología sociocultural, la arqueología y la lingüística. Finalmente, en lo referente a la psicología social, Worchel y Cooper (2002) apuntan que se relaciona con los fenómenos sociales para descubrir las leyes que rigen la convivencia de las personas; precisa que sus objetivos principales son el estudio del patrón de comportamiento del individuo -tanto en solitario como en grupo-, los roles que desempeñan y las situaciones que influyen en su conducta. 


\section{Actitudes del comportamiento organizacional}

La mayor parte de la investigación en co se interesa en tres actitudes (Robbins, 1998): 1) compromiso con el trabajo -el grado en que una persona identifica su trabajo, cómo participa activamente en él y cómo valora su propio desempeño-; 2) la satisfacción laboral -la actitud que cada trabajador o empleado tiene para con sus labores y los factores que intervienen en ellos-, y 3) el compromiso organizacional, que se refiere a cómo el empleado se identifica con la empresa y con sus objetivos propios, así como lo necesario para permanecer en ella o abandonarla. A fin de esquematizar lo correspondiente al co se presenta el cuadro 2. En este trabajo se aborda únicamente lo relativo a la sL, destacando a ésta como una actitud del co.

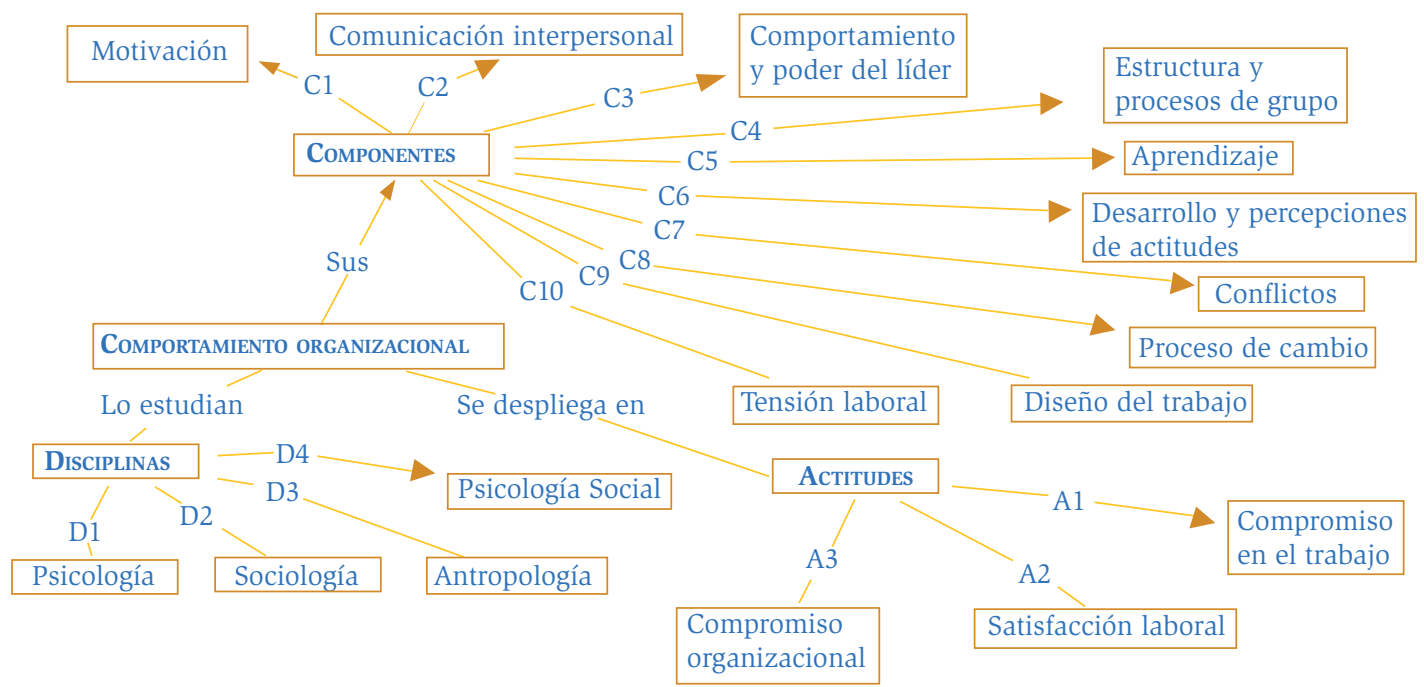

Fuente: Elaboración propia.

Figura 1. Elementos del comportamiento organizacional 


\section{Satisfacción laboral}

En cuanto al significado de sL, Sánchez expone que es "La sensación de bienestar derivada del cumplimiento de unas expectativas o de unas necesidades" (2008: 9), y explica que existe una clara diferencia entre esta variable y la motivación: la segunda implica energía y esfuerzo, por lo que se deriva de la actitud, y la primera pertenece al ámbito emocional. En la literatura académica fue posible identificar diversas variables observables contenidas en la sL, entre las que se encuentran: percepción económica, beneficios laborales, puesto, reconocimiento, relación con compañeros, supervisión del jefe, condiciones de trabajo y libertad de acción (cuadro 2).

\section{Cuadro 2. Variables contenidas en la satisfacción laboral}

VARIABLE DEFINICIÓN

Percepción económica Ingreso económico que recibe el trabajador. Es conMerino y Díaz (2008), Sán- secuencia de su esfuerzo, dedicación e índices guberchez (2008)

Beneficios laborales

Anaya y Suárez (1979), Sánchez (2008), Warr et al. (2008)

Puesto Abrajan, Contreras y Montoya (2008), Anaya (2005), Sánchez (2008) namentales y sindicales establecidos con anterioridad por la institución o empresa. En esta variable se incluyen también los incentivos y gratificaciones.

Incluye las promociones y ascensos que puede tener el empleado; aspectos relacionados con el tiempo -ya sea el horario de trabajo, el tiempo libre y el empleado en actividades diversas-, y la oportunidad de capacitarse o de incrementar el nivel de estudios.

Las condiciones en las que se encuentra el trabajador son primordiales para obtener un alto grado de sL. El lugar que ocupa en el organigrama puede ser un factor determinante para su pleno desarrollo, junto con las actividades propias del puesto. El diseño del puesto en relación con la personalidad del empleado es, sin duda, un factor determinante para mantener un nivel alto de sL. 


\section{Cuadro 2. Variables contenidas en la satisfacción laboral (Continuación)}

VARIABLE

Reconocimientos

Sánchez (2008), Tejero y

Fernández (2009)

\section{DEFINICIÓN}

Esta variable permite medir el grado de su del empleado por medio de los diversos tipos de reconocimiento que puede brindar la institución, ya sean escritos, económicos, sociales o culturales.

Relación con compañeros El ambiente de trabajo es fundamental para el buen Merino, Díaz y Runa desarrollo de las actividades. Al tener una relación (2008)

\section{Supervisión del jefe}

Benedito, Bonavia y Llinares (2008); Merino, Díaz y Runa (2008)

Condiciones de trabajo Molina et al. (2009)

\section{Libertad de acción}

Robbins y Judge (2009) cordial, amable, de comunicación y colaboración con los compañeros, la jornada laboral se hace más ligera, el empleado se desarrolla mejor y, como resultado, se obtienen más beneficios.

La interacción social cubre las necesidades del trabajo permitiendo tener un nivel alto de sL. La relación que el empleado tenga con su superior inmediato y con los altos mandos es esencial para el desarrollo del puesto, en beneficio de él mismo y de la empresa. De igual manera, un jefe que brinda retroalimentación, consejos y opiniones positivas demuestra interés por su personal.

Analiza el grado en que una persona se identifica y participa con su trabajo, así como el valor que le da a sus actividades. Se considera también que las características propias del mismo pueden resultar en un alto grado de sL.

Mide el grado de libertad e independencia que tiene el empleado para desarrollar su trabajo. La toma libre de decisiones, la aportación de opiniones sin miedo a represalias o indiferencia y la creación de su propia metodología de acción permiten al trabajador sentirse útil y realizado, lo que proporciona un alto grado de sL. 


\section{Factores socioculturales}

Con el término sociocultural se hace referencia a cualquier proceso o fenómeno relacionado con los aspectos sociales y culturales de una comunidad o sociedad (Bosque y Vilá, 1992), de tal modo que un elemento sociocultural tiene que ver exclusivamente con las realizaciones humanas que pueden ser útiles para organizar la vida comunitaria (Vilá, 1992) y para darle significado a la misma (Sánchez, 2005). En los estudios socioculturales por lo regular se utilizan conceptos como ideología, comunicación, etnicidad, clases sociales, estructuras de pensamiento, género, nacionalidad, medios de producción y muchos otros que sirven para comprender los elementos únicos de cada comunidad, sociedad y etnia (Bosque y Vilá, 1992; Nanda, 1987).

La relación del individuo con el entorno sociocultural se encuentra en continua retroalimentación, ya sea de forma positiva o negativa, y, dentro de los FSC, la educación es fundamental, ya que puede adoptar o ignorar factores tan diversos como el ambiental o el social (Díaz y Gallegos, 1996). El entorno y el medio ambiente brindan lo necesario para vivir; sin embargo, hay quienes opinan que el ser humano hace mal uso de los bienes de que dispone al dañar, por ejemplo, el medio ambiente (Hernández, 1996). En cuanto a la composición racial y étnica de una sociedad, la literatura (Otto, Sans y Montiel, 2003; Uribe, 2007) indica que determinadas conductas individuales, familiares, grupales 0 sociales influyen en el medio ambiente y, por ende, en la salud; de igual forma, en diversos estudios se relaciona a los FSC con ciertos padecimientos y enfermedades. Asimismo, los Fsc inciden en la valoración del trabajo, y hay evidencia de que los factores más asociados con el desempeño de las labores son la edad y la religión (Sastre, 2005). Algunas variables incluidas como FsC en los estudios sobre las instituciones de educación superior son: género, edad, religión, lugar de nacimiento, lugar de procedencia, estado civil, dependientes económicos, último grado de estudios, antigüedad en la institución, escuela de procedencia, estudios en curso, años de experiencia y si es egresado de la institución para la que labora (cuadro 3). 
Cuadro 3. Variables contenidas en los factores socioculturales

VARIABLE

Sexo

De la Maza y Armengol

(s.f.), Fabra y Camisón

(s.f.), Guzmán et al.

(2010), Sánchez, Fuen-

tes y Artacho (2007)

\section{Edad}

De la Maza y Armengol (s.f.), Fabra y Camisón

(s.f.), Guzmán et al. (2010), Lucas (s.f.)

\section{Religión}

Kreis y Brockopp

\section{Lugar de nacimiento}

\section{Estado civil}

Belfield y Harris

(2002), Clark (1991),

Fabra y Camisón (s.f.),

Nguyen, Taylor y Brad-

ley (2003)

\section{DEFINICIÓN}

Útil para realizar comparaciones y contrastar otros indi-

cadores como la edad, el estado civil, el último grado de estudios, etc. Se puede integrar en una hipótesis descriptiva, en una correlacional, en una de diferencia de grupos y/o en una causal.

Una de las relaciones que puede existir con la sL es la antigüedad en determinado puesto y la experiencia laboral.
Parte de la filosofía humanista permea todo su contexto, incluyendo al personal que labora a nivel académico y administrativo, a través de la difusión e interpretación de valores en los programas académicos y visión como IEs.

Su importancia radica en que tanto en México como fuera de él existe una variedad de factores culturales, sociales y de aprendizaje que influyen directamente en el comportamiento del individuo. Las instituciones educativas tienen una población multicultural, con personas nacidas dentro y fuera de la región, y se puede afirmar que cada institución presenta características diferentes.

Es uno de los factores que pueden marcar la diferencia en la sL dentro de las organizaciones.

Pueden encontrarse colaboradores solteros, casados, solteros o casados con hijos, casados sin hijos, etc., lo que en un momento determinado implica cuestiones de disponibilidad y desempeño. 


\section{Cuadro 3. Variables contenidas en los factores socioculturales}

(Continuación)

VARIABLE

Lugar de procedencia (años de estadía)

DEFINICIÓN

Que una persona provenga de cierto lugar u organización fuera de la región donde se vive tiene un impacto en la SL. Los colaboradores estarán identificados con diferentes prácticas laborales y métodos de trabajo. Lo anterior sustenta la relevancia de incluir una variable que ayude a desglosar este impacto.

Dependientes Se relaciona con el estado civil del individuo, pero existen económicos diferencias con base en las personas que dependen del colaborador. Esta situación repercute de manera directa en la SL.

Lugar de nacimiento Guzmán et al. (2010)
Suele marcar diferencia en el desarrollo de una organización, pues se considera una de las variables más trascendentales, y existen antecedentes que indican su influencia sobre la sL.

Antigüedad laboral en Se considera relevante sobre todo si hay un gran número la institución de trabajadores que han colaborado por mucho tiempo Lucas (s.f.) en la organización. También conviene detectar si algunos empleados han modificado su percepción de la sL dentro de la institución a través de los años.

Escuela de procedencia Determina si algunas instituciones tienen un mayor impacto en la sL, incluyendo a los egresados de las instituciones participantes. Además, ayuda a la generación de una idea más objetiva de la migración de colaboradores. 
Cuadro 3. Variables contenidas en los factores socioculturales

(Finaliza)

VARIABLE

DEFINICIÓN

Egresado de la misma institución
Ayuda a saber si existe una población mayoritaria de colaboradores egresados de algún programa académico de las mismas instituciones educativas. También permite tener un panorama más cercano de la perspectiva de SL que tienen sus colaboradores.

Indaga si el hecho de que los colaboradores se encuentren cursando algún programa académico contribuye a mejorar su sL. Asimismo, la actualización profesional es uno de los factores tangibles para incrementar la sL.
Años de experiencia profesional
Esta variable busca determinar si la experiencia docente y profesional de los colaboradores que integran el cuerpo académico/administrativo de las instituciones de educación afecta la visión de la organización. De igual modo, genera una idea más clara respecto al tipo de perfil (joven o maduro) del personal que labora en la institución.

\section{Factores ergoambientales}

La ergonomía ambiental estudia los factores ambientales que constituyen el entorno del sistema hombre-máquina, así como las condiciones físicas que lo rodean y que influyen en su desempeño al realizar diversas actividades. ${ }^{1}$ Entre estas condiciones se incluyen: ruido, iluminación, temperatura y vibración. En cuanto al ruido, la literatura académica (ACHs, 2008; Krüger y Zannin, 2004) 
indica que se puede caracterizar psicológicamente por resultar molesto e indeseable, físicamente por su aleatoriedad espectral y de intensidades, y desde el punto de vista de la comunicación por su bajo o nulo contenido informativo. La iluminación es un factor ambiental que interesa a los ergonomistas por su influencia en el desempeño de las tareas de los humanos; en general, puede interferir en la adecuada visualización de los objetos y entornos, en la eficiencia y eficacia del trabajador, en proporcionar la información adecuada y oportuna de señalización, además de que puede influir sobre el confort y la salud visual (Farrer et al., 1997; Hernández, 2006; Krüger y Zannin, 2004).

La tercera condición es la temperatura. Algunas investigaciones (Farrer et al., 1997; Krüger y Zannin, 2004) indican que para el ser humano es vital mantener y regular la temperatura interna del cuerpo, ya que, de forma similar a la materia, el organismo tiende a igualar su temperatura con el ambiente que lo rodea. La regulación térmica del cuerpo requiere un adecuado balance entre la cantidad de calor que produce el metabolismo y la actividad muscular y el calor que pierde hacia el ambiente, y el objetivo es mantener la temperatura interna entre 36 y $37^{\circ} \mathrm{C}$. Por último, con respecto a la vibración se afirma (Farrer et al., 1997; Krüger y Zannin, 2004) que el avance de la tecnología y su intervención en los puestos de trabajo han provocado que más trabajadores estén expuestos a vibraciones: en ocasiones estas no tienen consecuencias, pero a veces pueden afectar la salud y la capacidad de trabajo de quien se expone a ellas. Tales antecedentes indican que los pies, los glúteos y las manos son las áreas del cuerpo que comúnmente reciben y transmiten las vibraciones, dependiendo de la actividad que se realice y la posición en que se encuentre el operador.

En sus inicios, la ergonomía ambiental incluía la dimensión social, psicológica, cultural y organizacional de los entornos de los sistemas, pero ahora se enfoca más en los componentes individuales del ambiente físico (Parsons, 2000). Por ello, debe verse y practicarse desde su perspectiva más reciente, ya que las personas no responden de manera monótona a las medidas directas del ambiente físico, debido a sus características humanas que determinan tanto la sensibilidad como la respuesta a las mismas. La ergonomía ambiental es fundamental para el logro del bienestar y de la salud integral de los individuos, sin importar el contexto en que se encuentren. A continuación se presentan algunos antecedentes del estudio de esta variable en el contexto del sector educativo. 


\section{Satisfacción laboral y educación}

La satisfacción laboral, como lo señala Muñoz (1990), se estudia en todos los ambientes, jerarquías y puestos de trabajo, sin embargo, en este apartado se exponen investigaciones relacionadas únicamente con el sector educativo. Sobrado y Filgueira (1996) opinan que las particularidades del puesto y los factores personales tienen relación directa con la sL, y concluyen que el reconocimiento social, la responsabilidad del puesto, las relaciones con compañeros, alumnos y padres de familia y el sueldo son variables con gran repercusión sobre esta variable. En el Reino Unido, Johnson y Holdaway (1994) crearon y aplicaron la Técnica del Grupo Nominal; cuyos sujetos de estudio fueron directores de instituciones educativas. En su estudio teorizaron respecto de un modelo de SL (Mercer, 1997), recolectaron, por una parte, las características personales, contextuales y organizacionales, y por otra, aspectos de la percepción individual y personal del trabajador y su relación con otros individuos, y subrayaron la relevancia de los valores, su necesidad existencial y la percepción general de su ámbito.

Con sujetos de estudio similares a los de Johnson y Holdaway, Caballero y Salvador (2004) estimaron que $15 \%$ de los encuestados se sentía insatisfecho en un grado de moderado a alto, 31 \% reportaba satisfacción media y 54 \% satisfacción alta o muy alta; el total de la muestra estudiada fue de 1073 trabajadores. Por su parte, en 1993, Borg y Riding aplicaron una encuesta relacionada con el tema, y detectaron que de 97 directores y 53 jefes de departamento estudiados, solo 10 \% declaraba insatisfacción. No obstante, existen estudios (Cooper y Kelly, 1993) que reportan que la sL disminuye considerablemente entre los directivos de instituciones de educación superior y de escuelas primarias.

En la misma vertiente evaluatoria de la su en directores de escuelas, Armas (1996) agregó variables como el estrés y la autoestima; estudió 172 directivos, y como factores sobresalientes encontró el apoyo de los compañeros o del equipo de trabajo, el puesto ocupado y la responsabilidad asociada. Antes de este estudio, Bernal (1995) señaló que la insatisfacción laboral es consecuencia de la falta de integración de los profesores a los equipos. A su vez, Gunn y Holdaway $(1985,1986)$ encontraron que la sensación de logro, de realización y las relaciones con los compañeros son predictores asociados directamente con la sL. Un estudio efectuado con docentes de instituciones católicas en Madrid fue 
presentado por De Frutos et al. (2007), quienes reportan niveles de sL moderadamente satisfactorios, la inexistencia de signos de depresión, pero a la vez síntomas de ansiedad. Otros estudios (Anaya y Suárez, 2007) han identificado -respecto al diseño del trabajo- niveles medios y altos de sL en personal académico, sin embargo, en lo que respecta a la promoción, los superiores y el salario, los resultados muestran niveles medios de la variable de estudio. Algunos académicos (Falcón y Díaz, 2007) afirman que no hay diferencia en la sL entre el personal directivo femenino y masculino, ni entre los niveles educativos de los puestos que ocupan.

Como se observa en párrafos anteriores, los investigadores que han tratado el tema de la satisfacción laboral en directivos de instituciones de educación son heterogéneos. Cabe destacar que estos estudios se realizaron en diversos países (Armas, 1996; Bernal, 1995; Borg y Riding, 1993; Caballero y Salvador, 2004; Cooper y Kelly, 1993; Falcón y Díaz, 2007; Gunn y Holdaway, 1985, 1986; Mercer, 1993; Sobrado y Filgueira, 1996; Tejero y Fernández, 2009).

\section{Factores socioculturales y educación}

Los Fsc se han examinado a partir de y en el sector educativo. Algunas investigaciones se detallan a continuación. Ramírez, Cubero y Santamaría (1990) analizan el impacto de los Fsc en el aprendizaje de adultos. Su estudio se realizó en un grupo de mujeres entre los 35 y los 55 años en una comunidad de Sevilla, con niveles académicos que oscilaban entre analfabetas, pregraduadas y graduadas. Davies (2006) aplicó una encuesta para determinar el impacto de los Fsc en las personas encargadas de realizar los planes de estudio y contenidos temáticos, y encontró que existe un gran conflicto entre estos factores y las decisiones por tomar respecto a los temas de las materias. Destaca la opinión de los alumnos en cuanto a los resultados.

Dos años de análisis y observaciones a estudiantes internacionales de primer grado fueron necesarios para determinar si los Fsc repercutían tanto en su relación con otros compañeros como en su desarrollo humano. Gu, Schweisfurth y Day (2010) encontraron que, pese a que provenían de diferentes países y aparentemente hablaban el mismo idioma, la comunicación con los demás era difícil, además, detectaron que las políticas administrativas de la institución complicaron el camino, y cuando no se logró la adaptación se registró un 
bajo aprovechamiento académico. Kenneth y Chan (2004) llevaron a cabo una investigación entre las diferentes etnias, edades y género de los estudiantes que cursaban el nivel básico en un país africano. El porcentaje mayor era de estudiantes blancos, y el resto, negros, pero en condiciones de igualdad social; sin embargo, el logro académico fue más elevado en las estudiantes blancas, y entre los estudiantes de color se observó un alto índice de deserción.

\section{Factores ergoambientales y educación}

La ergonomía ambiental también es relevante en materia de bienestar y salud integral de los individuos. A continuación se presentan algunos estudios llevados a cabo en el contexto educativo. En la investigación de Krüger y Zannin (2004) se exponen resultados acerca del funcionamiento de los salones de clase en una institución de educación superior. Estos autores consideraron tres de los cuatro factores ergoambientales -excluyeron la vibración, dada su falta de significancia en el contexto estudiado.

Para determinar la eficacia de un programa que cumpliera con los estándares en ergonomía ambiental dentro de una universidad, Bidassie et al. (2010) analizaron todas las instalaciones para detectar carencias al respecto; sus resultados pusieron de manifiesto que las carencias en los FEA tenían un costo muy alto, y estos debieron haber sido evaluados desde la construcción del plantel. Los cambios significativos para mejorar la iluminación y la temperatura son excesivos. Al final, proponen que en la construcción de cualquier institución, y más si se trata de una educativa, el diseño se realice conjuntamente con los expertos en ergonomía.

Gálvez y Ponce (2005) indagaron las condiciones de riesgo ergonómico a las que se exponen los profesores en una institución educativa; relacionaron los puestos de trabajo y la antigüedad con diversas patologías, como el síndrome del túnel carpiano, tendinitis, cervicalgia y lumbalgia. A los trabajadores con alguno de estos trastornos se les brindó tratamiento especializado, y se revisaron las posibilidades de cambiarlos de puesto o de tareas.

Así, en el diseño de nuevos modelos de representación de información en formato electrónico está implícita la ergonomía. Resulta necesario identificar los FEA en el esquema de herramientas visuales que ayudan al usuario a no tener padecimientos de la vista, como consecuencia de la falta de luz. Hernández 
(2006) propone la construcción de modelos virtualmente educativos a partir de la ergonomía.

\section{Modelo conceptual propuesto}

Basados en los antecedentes académicos expuestos, se propone un modelo conceptual (figura 2) en el que se indican las relaciones entre las variables que conforman los FSC y los FEA, así como su relación con la sL.

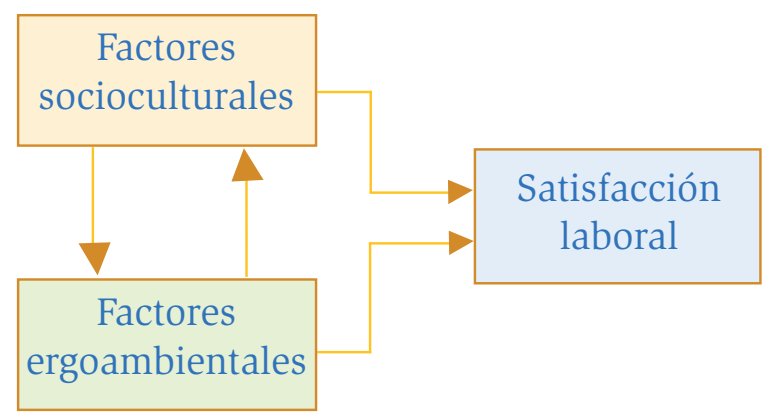

Fuente: Elaboración propia.

Figura 2. Marco conceptual propuesto

Para este modelo, es necesario determinar cuál o cuáles variables de cada constructo tienen mayor o menor relación con el resto de los indicadores y, a su vez, establecer aquellas que tienen algún impacto sobre la satisfacción laboral. En la figura 2 se presentan tres variables latentes cuyas variables observables se detectaron en estudios previos -de acuerdo con la revisión exhaustiva de la literatura del ramo-, así como en otros indicadores que se consideran relevantes para el análisis del objeto de estudio. Las variables observables se detallan a continuación de manera gráfica (figuras 3, 4 y 5). 


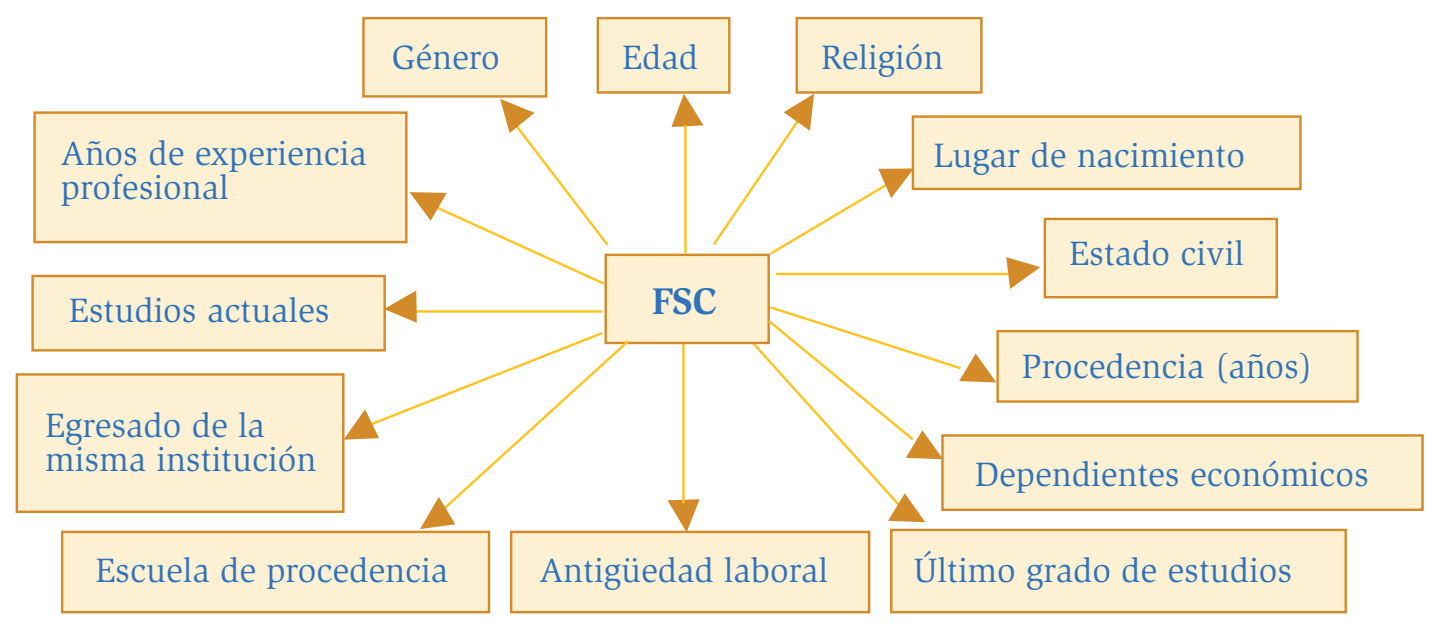

Fuente: Elaboración propia.

Figura 3. Indicadores del constructo factores socioculturales

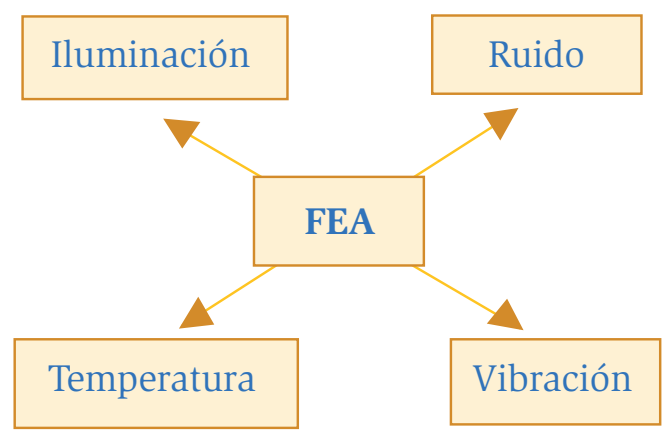

Fuente: Elaboración propia.

Figura 4. Indicadores del constructo factores ergoambientales 


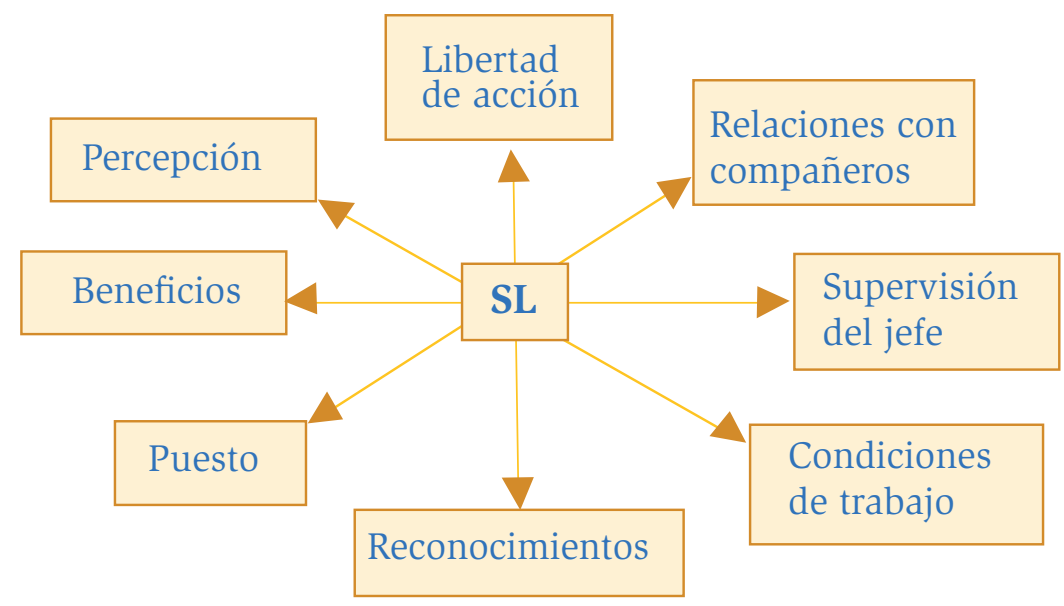

Fuente: Elaboración propia.

Figura 5. Indicadores del constructo satisfacción laboral

\section{Conclusiones y recomendaciones}

Con base en la revisión de la literatura de los temas satisfacción laboral, factores socioculturales y ergoambientales, y en el análisis de diferentes investigaciones, se identificaron variables relevantes para integrar un modelo de medición y proponer un modelo conceptual respecto de la relación entre los factores socioculturales y los ergoambientales, y su impacto en la satisfacción laboral en las instituciones de educación superior en México.

Se recomienda la aplicación empírica de este modelo, a fin de determinar las variables más representativas de los factores socioculturales y ergoambientales y el impacto de estos sobre la su en diversos ámbitos y niveles educativos -instituciones públicas o privadas, desde el preeescolar hasta la universidad-. Puesto que se plantea un modelo multivariante, se aconseja la utilización de modelos de ecuaciones estructurales, ya que estos permiten analizar el efecto conjunto de las distintas variables (Jöreskog, 1993) y determinar tanto su impacto como la posible relación entre ellas. 


\section{Fuentes consultadas}

Abrajan, M., J. Contreras y S. Montoya (2009). "Grado de satisfacción laboral y condiciones de trabajo: una exploración cualitativa”. Enseñanza e Investigación en Psicología, 14 (1), enero-junio, 105-118.

ACHS (2008). "Ergonomía ambiental, niveles de confort” [en línea]. Asociación Chilena de Seguridad. Disponible en:

http://www.inp.cl/portal/Documentos/prevencion/guia_ergonomia_ambiental.pdf y en: http://transparencia-activavm.

inp.cl/portal/Documentos/prevencion/guia_ergonomia_ambiental.pdf

Anaya, D. y J. Suárez (2007). "Satisfacción laboral de los profesores de educación infantil, primaria y secundaria. Un estudio de ámbito nacional”. Revista de Educación, 344, 217-243.

Armas, M. (1996). "Evaluación de la satisfacción laboral, estrés y autoestima de los directivos escolares", en Dirección participativa y evaluación de centros. Actas del II Congreso Internacional sobre Dirección de Centros Docentes. Bilbao: Instituto de Ciencias de la Educación de la Universidad de Deusto, 419-429.

Ballesteros, S. (1994). Cognitive Approaches to Human Perception. Hillsdale: Erlbaum.

Belfield, C. y R. Harris (2002). "How well do Theories of Job Matching Explain Variations in Job Satisfaction across Education Levels? Evidence for UK Graduates”. Applied Economics, 34, 535-548.

Benedito, M., T. Bonavia y L. Llinares (2008). "Relación entre las prioridades de valor y la satisfacción laboral”. Revista Colombiana de Psicología, 17, 59-73.

Bernal, J. (1995). "La satisfacción de los directivos con su trabajo: su autoestima, organización y gestión educativa”. Relieve, 5, 3-7.

Bersanelli, V. (1979). Manual de psicología. México: Editorial Técnica.

Bidassie, B. et al. (2010). "Limited Economic Evaluation to Assess the Effectiveness of a University-Wide Office Ergonomics Program”. Applied Ergonomics, 41 (3), 417-427.

Borg, M. y R. Riding (1993). "Occupational Stress and Job Satisfaction among School Administrators”. Journal of Educational Administration, 31 (1), $4-21$. 
Bosque, J. y J. Vilá (1992). Geografía de España, geografía humana. Barcelona: Planeta.

Brunner, J. (1990). Educación superior en América Latina: Cambios y desafíos. Santiago de Chile: Fondo de Cultura Económica.

Burgos, J. (2005). Antropología: Una guía para la existencia. Madrid: Palabra. Caballero, J. y F. Salvador (2004). "Satisfacción e insatisfacción de los directores escolares”. Revista de Educación, 333, 363-384.

Cisneros, P. (2006). "El proceso del cambio en el comportamiento organizacional”. Pshike, 3 (8).

Clark, B. (1991). El sistema de educación superior: Una visión comparativa de la organización académica. México: Nueva Imagen/Universidad Autónoma Metropolitana/Universidad Futura.

Davies, L. (2006). “Global Citizenship: Abstraction or Framework for Action?”. Educational Review, 58 (1), 5-25.

De Frutos, J. et al. (2007). Condiciones de trabajo y salud laboral de los docentes en el ámbito de las Escuelas Católicas de Madrid. Madrid: Centro de Enseñanza Superior en Humanidades y Ciencias de la Educación "Don Bosco"/Escuelas Católicas de Madrid/Federación de Sindicatos Independientes de Enseñanza-Madrid.

De la Maza, M. y A. Armengol (s.f.). "Trastornos alimentarios: Anorexia y bulimia como autoagresión”. Revista de Psicología y Salud, 15 (4).

Díaz, O. y R. Gallegos (1996). Formación y práctica docente en el medio rural. México: Plaza y Valdés.

Donolo, C. et al. (1985). La cultura del 900. 4. Sociología, economía, derecho, historiografía. México: Siglo xxi Editores.

Fabra, M. y C. Camisón (s.f.). "Ajuste entre el capital humano del trabajador y su puesto de trabajo como determinante de la satisfacción laboral". Revista del Ministerio de Trabajo e Inmigración, 76, 129-142.

Fuentes, R. (2001). "Conflictos y negociaciones del comportamiento organizacional en el sector educativo de Chile”. Administración, 12 (3).

Gálvez, L. y M. Ponce (2005). "Condiciones de riesgo ergonómico en los administrativos de una institución de educación superior en Pereira”. Investigaciones Andinas, 7 (10), 5-9.

104 Gilbert, J. (1997). Introducción a la sociología. Santiago de Chile: Lom Ediciones. 
González, M., A. Hernández de Rincón y A. Hernández (2007). “Desempeño de alumnos y docentes de matemática desde el constructivismo. Caso Álgebra Lineal. Facultad de Ingeniería. LUZ”. Multiciencias, 7 (3), 282292.

Gordon, J. (1997). Comportamiento organizacional. México: Prentice-Hall. Gu, Q., M. Schweisfurth y Ch. Day (2010). “Learning and Growing in a 'Foreign' Context: Intercultural Experiences of International Students”. Compare, 40 (1), 7-23.

Gunn, J. y E. Holdaway (1985). “Principal Job Satisfaction”. Canadian Administrator, 24 (7), 1-5.

------ (1986). "Perceptions of Effectiveness, Influence and Satisfaction of Senior High School Principals”. Educational Administration Quarterly, 22 (2), 43-62.

Guzmán, T. et al. (2010). "La satisfacción laboral del capital humano femenino en la actividad hotelera. Un estudio de caso para Andalucía y Algarve”. Revista Universitaria de Ciencias del Trabajo, 11, 127-145.

Hernández, A. (2006). "Principios ergonómicos aplicados a los mapas de conocimiento: ventajas y desventajas de las nuevas formas de representación de la información”. Acimed, 14 (3).

Hernández, P. (1996). Políticas demográficas y factores socioculturales: análisis de sociología política. Washington: Pan American Union.

Herzberg, F. (1966). Work and the Nature of Man. Nueva York: The World Publishing Company.

Ivancevich, J., R. Konopaske y M. Matteson (2006). Comportamiento organizacional. 7a ed. México: Prentice-Hall.

Johnson, N. y E. Holdaway (1994). "Factor Importance and Job Satisfaction of School Principals”. British Educational Research Journal, 20, 17-33.

Jones, E. (1990). Interpersonal Perception. Nueva York: W.H. Freeman.

Jones, G. (2008). Teoría organizacional, diseño y cambio en las organizaciones. 5a ed. México: Prentice-Hall.

Jöreskog, K. (1993). Modelado de ecuaciones estructurales. Zarautz: Instituto Vasco de Estadística.

Kenneth, T. y F. Chan (2004). "Social Competence in Zimbabwean Multicultural Schools: Effects of Ethnic and Gender Differences”. International Jour- 
nal of Psychology, 39 (3), 169-178.

Kreis, K. y D. Brockopp (1986). “Autonomy: A Component of Teacher Job Satisfaction”. Education, 107 (1), 110-115.

Krüger, E. y P. Zannin (2004). "Acoustic, Thermal and Luminous Comfort in Classrooms”. Building and Environment, 39, 1055-1063.

Locke, E. (1978). "The Ubiquity of Technique of Goal Setting in Theories of and Approaches to Employee Motivation". Academy of Management Review, 3, 594-601.

Lucas, A. (s.f.). "Una aproximación sociológica al estudio de la satisfacción en el trabajo en la provincia de Santander”. Reis. Revista Española de Investigaciones Sociológicas, 13 (81), 65-100.

Marginson, S. (2009). "The Knowledge Economy and Higher Education: A System for Regulating the Value of Knowledge". Innovación Educativa [en línea], 9 (47), 63-71. Disponible en: http://www.redalyc.org/articulo. oa? id $=179414895007$

Merino, M. y A. Díaz (2008). "El estudio del nivel de satisfacción laboral en las empresas públicas y privadas de Lambayeque”. Hatun Runa [en línea], 1, 132-142. Disponible en: http://dialnet.unirioja.es/servlet/ articulo? codigo $=2710495$

Miner, J.B. (2003). “The Rated Importance, Scientific Validity, and Practical Usefulness of Organizational Behavior Theories: A Quantitative Review”. Academy of Management Learning \& Education, 2 (3), 250-268.

Mitchell, T. (1997). "Matching Motivational Strategies with Organizational Contexts". Research in Organizational Behavior, 19, 60-62.

Molina, J. et al. (2009). "Factores relacionados con la satisfacción laboral de enfermería en un hospital médico-quirúrgico". Investigación y Educación en Enfermería, 28 (2), 218-225.

Moreno, D., M. Cepeda y P. Romero (2004). “El modelo de evaluación, intervención y análisis de procesos como propuesta de diseño instruccional”. Enseñanza e Investigación en Psicología [en línea], 9 (2), 271-291. Disponible en: http://www.redalyc.org/articulo.oa?id = 29290205

Muñoz, A. (1990). "Satisfacción e insatisfacción en el trabajo”. Tesis doctoral. Madrid: Universidad Complutense de Madrid.

106 Nanda, S. (1987). Antropología cultural, adaptaciones socioculturales. México: 
Grupo Editorial Iberoamérica.

Nguyen, A., J. Taylor y S. Bradley (2003). Relative Pay and Job Satisfaction: Some New Evidence. Lancaster: Lancaster University Management School (Working Paper 45).

osh (2011). “Organización de Seguridad y Salud. Diseño del trabajo” [en línea]. Disponible en: http://www.osh.net/directory/legal/legal_07.htm

Otto, I., M. Sans y M. Montiel (2003). "Necesidad de nutrición y eliminación”. Masson, 64-78.

Parsons, K.C. (2000). "La ergonomía ambiental: Una revisión de principios, métodos y modelos”. Applied Ergonomics, 31 (6), 581-594.

Ramírez, J., M. Cubero y A. Santamaría (1990). “Cambio sociocognitivo y organización de las acciones: Una aproximación sociocultural a la educación de adultos". Infancia y Aprendizaje, 51-52, 169-190.

Robbins, S. y T. Judge (2009). Comportamiento organizacional. 13a ed. México: Prentice Hall.

Robles, M. et al. (2005). "Variables relacionadas con la satisfacción laboral: un estudio transversal a partir del modelo EFQm”. Gaceta Sanitaria, 19 (2), 127-134.

Rueda, M., L. Elizalde y A. Torquemada (2003). "La evaluación de la docencia en las universidades mexicanas”. Revista de la Educación Superior [en línea], 127, 71-77. Disponible en: http://publicaciones.anuies.mx/ revista/127/3/5/es/la-evaluacion-de-la-docencia-en-las-universidadesmexicanas

Sánchez, C. (2008). "Motivación, satisfacción y vinculación. ¿Es gestionable la voluntad de las personas en el trabajo?”. Acción Psicológica, 5 (1), 9-28.

Sánchez, S., F. Fuentes y C. Artacho (2007). "La satisfacción laboral desde la perspectiva de género. Un análisis empírico mediante modelos logit y probit”, en J.C. Ayala Calvo (coord.), Conocimiento, innovación y emprendedores: camino al futuro. La Rioja: Universidad de La Rioja, 3445-3457.

Sastre, V. (2005). La cultura del ocio. Implicaciones sociales y eclesiásticas del fin de semana. Madrid: Universidad Pontificia Comillas de Madrid.

Sobrado, L. y L. Filgueira (1996). "Diagnóstico psicopedagógico de la satisfacción profesional de los directores de centros docentes”, en Dirección 
participativa y evaluación de centros. Actas del II Congreso Internacional sobre Dirección de Centros Docentes. Bilbao: Instituto de Ciencias de la Educación de la Universidad de Deusto, 621-630.

Tejero, C. y M. Fernández (2009). "Medición de la satisfacción laboral en la dirección escolar”. Relieve [en línea], 15 (2), 1-16. Disponible en: http:// www.uv.es/RELIEVE/v15n2/RELIEVEv15n2_1.htm

Uribe, J. (2007). Anorexia: los factores socioculturales de riesgo. Medellín: Universidad de Antioquía.

Wang, J. et al. (2009). "Changes in Perceived Job Strain and the Risk of Major Depression: Results From a Population-Based Longitudinal Study”. American Journal of Epidemiology, 169 (9), 1085-1091.

Warr P., J. Cook y T. Wall (1979). "Scales for the Measurement of Some Work Attitudes and Aspects of Psychological Well-Being". Journal of Occupational Psychology, 52, 129-148.

Worchel, S. y J. Cooper (2002). Psicología social. Connecticut: Cengage Learning. 